\title{
Systematic study on the mechanical and electric behaviors of the nonbuckling interconnect design of stretchable electronics
}

\author{
Hao Liu ${ }^{1,2}$, RiYe Xue ${ }^{3}$, JianQiao $\mathrm{Hu}^{2}$, XueCheng Ping ${ }^{4}$, HuaPing $\mathrm{Wu}^{5}$, MingQi Huang ${ }^{3}$, \\ Han Zhang ${ }^{6}$, Xu Guo ${ }^{3}$, Rui $\mathrm{Li}^{3^{*}}$, YuLi Chen ${ }^{1^{*}}$, and YeWang $\mathrm{Su}^{2,7,8,9^{*}}$ \\ ${ }^{1}$ Institute of Solid Mechanics, Beihang University, Beijing 100191, China; \\ ${ }^{2}$ State Key Laboratory of Nonlinear Mechanics, Institute of Mechanics, Chinese Academy of Sciences, Beijing 100190, China; \\ ${ }^{3}$ State Key Laboratory of Structural Analysis for Industrial Equipment, Department of Engineering Mechanics, and International Research \\ Center for Computational Mechanics, Dalian University of Technology, Dalian 116024, China; \\ ${ }^{4}$ School of Mechanical Engineering, Tianjin University of Science \& Technology, Tianjin 300222, China; \\ ${ }^{5}$ Key Laboratory of E\&M (Zhejiang University of Technology), Ministry of Education \& Zhejiang Province, Hangzhou 310014, China; \\ ${ }^{6}$ Institute of Acoustics, Chinese Academy of Sciences, Beijing 100190, China; \\ ${ }^{7}$ School of Engineering Science, University of Chinese Academy of Sciences, Beijing 100049, China; \\ ${ }^{8}$ State Key Laboratory of Structural Analysis for Industrial Equipment, Department of Engineering Mechanics, Dalian University of \\ Technology, Dalian 116024, China; \\ ${ }^{9}$ State Key Laboratory of Digital Manufacturing Equipment and Technology, Huazhong University of Science and Technology, \\ Wuhan 430074, China
}

Received March 26, 2018; accepted May 4, 2018; published online September 5, 2018

\begin{abstract}
Recently, we developed a nonbuckling interconnect design that provides an effective approach to simultaneously achieving high elastic stretchability, easiness for encapsulation, and high electric performance for stretchable electronics. This paper aims to systematically study its mechanical and electric behaviors, including comparisons of the nonbuckling and buckling interconnect designs on stretchability, effects of the thickness on electric performance, and modeling and experimental investigations on the finite deformation mechanics. It is found that the results on stretchability depend on the layouts. Long straight segments and small arc radii for nonbuckling interconnects yield an enhancement of stretchability, which is much better than that of buckling designs. On the other hand, shorter straight segments or thicker interconnects are better to lower the resistances of interconnects. Therefore, optimization of the designs needs to balance the requirements of both the mechanical and electric performances. The finite deformation of interconnects during stretching is analyzed. The established analytic model is well validated by both the finite element modeling and experimental investigations. This work is key for providing the design guidelines for nonbucklingbased stretchable electronics.
\end{abstract}

nonbuckling, finite deformation, stretchability, stretchable electronics

PACS number(s): $62.20 .-\mathrm{x}, 46.05 .+\mathrm{b}, 85.40 .-\mathrm{e}$

Citation: H. Liu, R. Y. Xue, J. Q. Hu, X. C. Ping, H. P. Wu, M. Q. Huang, H. Zhang, X. Guo, R. Li, Y. L. Chen, and Y. W. Su, Systematic study on the mechanical and electric behaviors of the nonbuckling interconnect design of stretchable electronics, Sci. China-Phys. Mech. Astron. 61, 114611 (2018), https://doi.org/10.1007/s11433-018-9238-2

*Corresponding authors (YeWang Su, email: yewangsu@imech.ac.cn; Rui Li, email:

ruili@dlut.edu.cn; YuLi Chen, email: yulichen@buaa.edu.cn) 


\section{Introduction}

As an emerging class of technology, stretchable electronics has received considerable attention [1]. With the rapid development of materials, structural designs, and manufacturing techniques, an increasing number of potential applications have been developed, such as hemispherical electronic eye cameras [2,3], epidermal electronics [4], fingertip electronics [5,6]), stretchable batteries [7], and soft robotics [8].

It is known that flexible electronics has planar thin film configurations while the target surfaces, such as the clothes, skins, and organs to which the electronics is attached, have various complex shapes and are usually time-varying; thus, to achieve high-performance bio-electronic devices, there is a key demand for dynamic conformal contact of the electronics with arbitrary surfaces. Figure 1(a) and (b) illustrates the necessity of stretchability by sketching a thin film that fits onto a developable surface, represented by a cylinder (Figure 1(a)), and a non-developable surface, represented by a sphere (Figure 1(b)), respectively. It is seen that the film can be flattened onto a cylinder (developable surface) without stretching, but this is impossible on a sphere (nondevelopable surface), where wrinkling is inevitable unless the film is properly stretched. Therefore, perfect fit with the most common non-developable surfaces requires that the electronic devices be stretchable, not just flexible (e.g., foldable and bendable), as illustrated in the preceding paragraph, where the elastic stretchability is of crucial importance for all of the listed electronic systems.

Existing structural designs (Figure 1(c)) for interconnects that can offer stretchability in electronic devices have experienced an evolution from straight [2,9-11] to curvilinear layouts $[11,12]$, from bonding to or embedding in the supporting substrates [13] to free-standing configuration [14], and from simple [11] to self-similar fractal structures [7,1518]. It should be noted that, although stretchability is effectively improved, the same strategy has been applied to all above schemes, i.e., out-of-plane buckling of thin interconnects, as shown in Figure 1(c). Some important advances in this respect have been recently made, such as the buckling of serpentine microstructures [19], postbuckling of fractal interconnects partially bonded to a substrate [20], and theory of large-displacement space curved beams accounting for geometric nonlinearity and different scalings of kinematic variables [21]. The limitations of the above systems are at least twofold. First, the freestanding interconnects bring a challenge for encapsulation of practical stretchable electronics, which usually induces much reduction in stretchability. The maximum stretchability is only $\sim 60 \%$ for the bonded interconnects without prestrain; this corresponds only to $\sim 12 \%$ system stretchability under $\sim 64 \%$ areal coverage of active devices [22]. Second, the buckled interconnects with thin-film configuration cause high electric resistances; this brings about high power dissipation and heat generation, thus leading to poor performance and reliability of the devices such as stretchable arrays of LEDs.

To address the problems in out-of-plane buckling designs, Su et al. [17] introduced a nonbuckling design, providing a novel route to more advanced stretchable electronics. Figure 1(c) shows the experimental and finite element modeling results for an $80-\mu \mathrm{m}$-thick nonbuckling copper serpentine interconnect that is bonded to an Ecoflex substrate, which can achieve $96 \%$ stretchablity. Further study shows that the nonbuckling design for metal materials can achieve the stretchability as large as $\sim 350 \%$ (around six times larger than $\sim 60 \%$ stretchablity by the buckling design), and $\sim 90 \%$ stretchability can be realized even for brittle inorganic semiconductor materials such as silicon [17]. With the thickness properly increased, the nonbuckling serpentine interconnects can simultaneously implement high elastic (a)

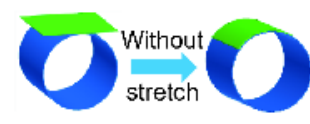
surface

\section{(c)}

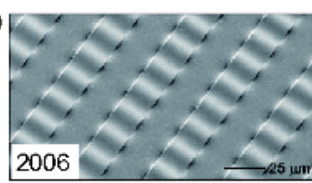

(b)

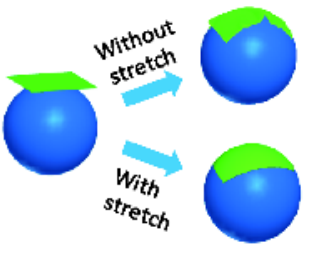

Non developable surface

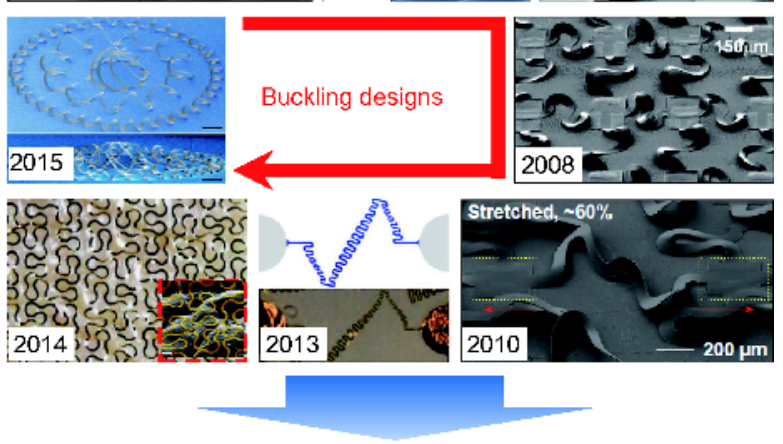

Non buckling design

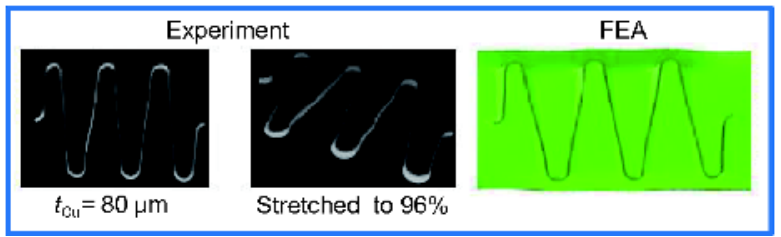

Figure 1 (Color online) Schematic illustrations of a thin film fitting onto (a) a developable surface represented by a cylinder and (b) a non-developable surface represented by a sphere. (c) Evolution of the buckling designs for interconnects $[7,9-12,15,18]$ as well as the experiment and finite element modeling [17] for an $80-\mu \mathrm{m}$-thick nonbuckling copper serpentine interconnect that is bonded to an Ecoflex substrate when stretched to $96 \%$ applied strain. Reproduced with permission from refs. [7,9-12,15,17,18]. 
stretchability, easiness for encapsulation, and high electric performance (with low resistances, power dissipation and heat generation) [17]. A few recent advances in this respect have been reported, such as the investigations on stretchability and compliance of freestanding serpentine-shaped ribbons [23], finite deformation model of planar serpentine interconnects [24], and elasticity solutions to nonbuckling wide serpentine ribbons [25]. However, there still exists a pending puzzle of extreme importance: does the nonbuckling design always win on stretchability?

With the key problem being a start, this paper aims to systematically study the mechanical and electric behaviors of nonbuckling interconnects, including comparisons of the nonbuckling and buckling interconnect designs on stretchability, effects of the thickness on electric performance, and modeling and experimental investigations on the finite deformation mechanics. Typical serpentine interconnects that are bonded to a compliant elastomeric substrate are systematically investigated by the finite element modeling, where different deformation modes are explored, including both the buckling and nonbuckling modes. By analyzing the impact of the straight segment lengths and arc radii on stretchability, we summarize the guidelines for a nonbuckling-based system to achieve favorable stretchability and suggest the scope of applicability of the nonbuckling design for stretchable electronics. An elastica theory-based analytic finite deformation model is then established, by which we obtain accurate mechanical behaviors of nonbuckling interconnects. Comprehensive finite element analysis (FEA) and experimental investigations are carried out, which validate the analytic model. The current work provides a basis for refined nonbuckling design toward high-performance stretchable electronics.

\section{Systematic comparisons of the buckling and nonbuckling interconnects on the mechanical stretchability and electric performance}

A class of typical serpentine interconnects are studied by FEA in this section, with the computational model illustrated in Figure 2(a), where the $\mathrm{Cu}$ interconnect with a fixed width of $50 \mu \mathrm{m}$ is bonded to a $0.5-\mathrm{mm}$-thick Ecoflex substrate. The stretchability is examined by changing the thickness of $\mathrm{Cu}$ interconnect, $t_{\mathrm{Cu}}$, in a wide range, from $10 \mathrm{~nm}$ to $500 \mu \mathrm{m}$. Due to symmetry, only a half period of the interconnect has been analyzed in the commercial software package ABAQUS [26], with displacement loading applied in the direction of stretching. The 8-node linear brick element was adopted for the Ecoflex substrate that was regarded as a hyperelastic material as described by the Mooney-Rivlin model, with $\mathrm{C}_{10}=0.008054 \mathrm{MPa}, \mathrm{C}_{01}=0.002013 \mathrm{MPa}$, and $\mathrm{D}_{1}=2.0 \mathrm{MPa}^{-1}$.
The 4-node doubly curved thin or thick shell element was adopted for the interconnect that was described by an elasticperfectly plastic material, with the Young's modulus $124 \mathrm{GPa}$, Poisson's ratio 0.34, and yield stress $372 \mathrm{MPa}$.

Take the black curve in Figure 2(b) and corresponding deformation modes in Figure 2(c) as an example; this corresponds to the interconnect with arc radius $R=0.25 \mathrm{~mm}$ and straight segment length $L=3 \mathrm{~mm}$. It is interesting to see that, with increasing the $\mathrm{Cu}$ thickness, the elastic stretchability of the system increases, then decreases, and increases again. The wrinkling deformation mode is yielded for the $\mathrm{Cu}$ with nano-scale thicknesses, and thicker $\mathrm{Cu}$ induces larger wavelength, as shown in Figure $2(\mathrm{c})$. When $t_{\mathrm{Cu}} \approx 50 \mu \mathrm{m}$, a transition from the wrinkling/buckling deformation mode to nonbuckling mode is detected from FEA. For thicker $\mathrm{Cu}$, the nonbuckling mode dominates the deformation of the interconnects; this is also called scissoring mode as the deformed regime is like scissors. The quantitative results in Figure 2(b) confirm that the nonbuckling design gives better stretchability over buckling designs. The elastic stretchability under the nonbuckling mode is as large as $\sim 120 \%$, while the optimal stretchability of buckling designs is only $\sim 30 \%$.

The effect of the length $L$ of straight segments on

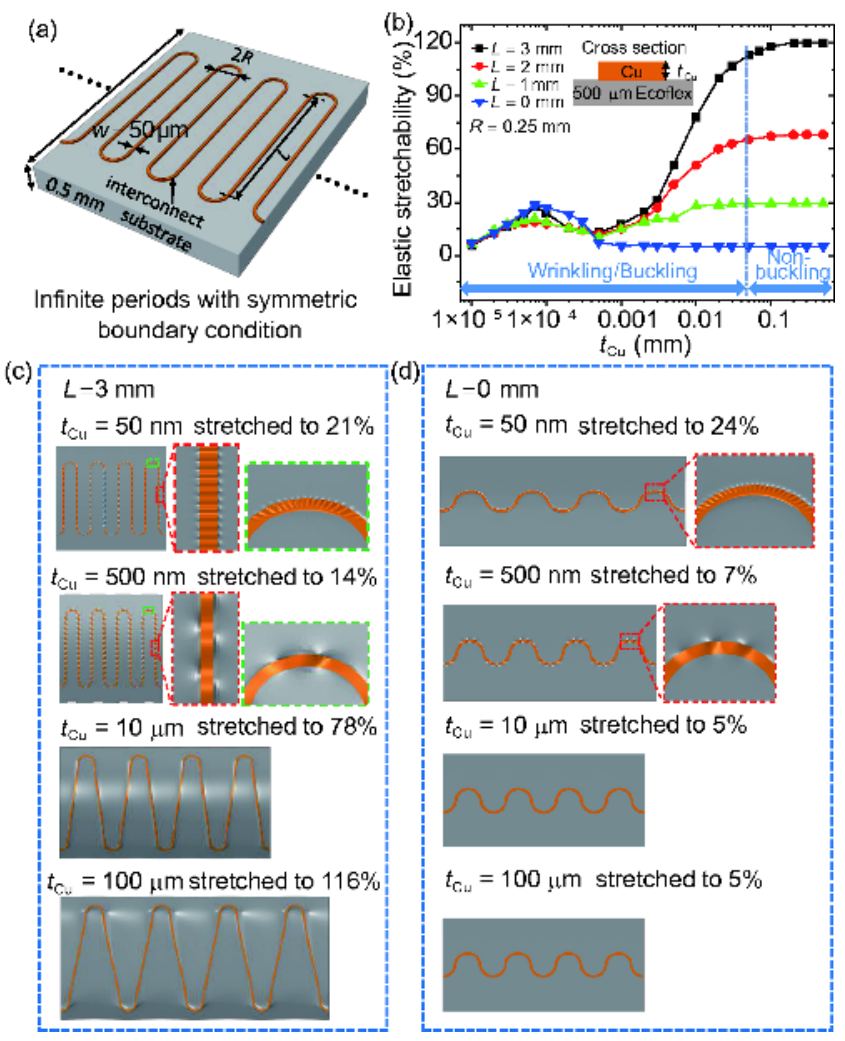

Figure 2 (Color online) (a) Computational model of a typical serpentine interconnect that is bonded to a substrate; (b) elastic stretchability versus the thickness of $\mathrm{Cu}$ interconnects with $R=0.25 \mathrm{~mm}$ and $L=0,1,2$, and $3 \mathrm{~mm}$; observation of different deformation modes for the interconnects having (c) $L=3 \mathrm{~mm}$ and (d) $L=0$, with $t_{\mathrm{Cu}}=50 \mathrm{~nm}, 500 \mathrm{~nm}, 10 \mu \mathrm{m}$, and $100 \mu \mathrm{m}$. 
stretchability is studied with $R=0.25 \mathrm{~mm}$ fixed, as shown in Figure 2(b). The blue, green, red, and black curves correspond to $L=0,1,2$, and $3 \mathrm{~mm}$, respectively. There is no obvious law found for the optimal stretchability for buckling designs, but for the nonbuckling design, longer straight segments provide significantly larger stretchability. As the straight segment length $L$ increases from 0 to $3 \mathrm{~mm}$, the stretchability increases from $\sim 5 \%$ to $\sim 120 \%$. The case with $L$ $=1 \mathrm{~mm}$ (green curve) is almost the threshold, of which the nonbuckling design-based stretchability approaches the optimal one of buckling designs. For $L>1 \mathrm{~mm}$, the nonbuckling design is able to provide larger stretchability, while buckling designs do for $L<1 \mathrm{~mm}$. It is worth pointing out that, with increasing $t_{\mathrm{Cu}}$, the stretchability of the system without straight segments in the interconnect only experiences an increase and then a decrease, but without any more increase. The stretchability reaches saturation at a low level ( $5 \%)$, no matter how large $t_{\mathrm{Cu}}$ is, as indicated by the blue curve and Figure 2(d). These results clearly show that whether the nonbuckling design wins on stretchability depends on the interconnect layouts.

We further examine the effects of the arc radius $R$ by reducing it to 0.2 and $0.15 \mathrm{~mm}$, as plotted in Figure 3(a) and (b), respectively. Stretchability versus the thickness and straight segment length of the $\mathrm{Cu}$ interconnect shows the same trend as that in Figure 2(b) for $R=0.25 \mathrm{~mm}$, thus validating the findings derived from Figure 2. It is found that, with a fixed $L$, smaller $R$ yields larger stretchability for the nonbuckling design. For example, $R=0.15 \mathrm{~mm}$ gives the largest stretchability as high as $\sim 160 \%$, as compared with $\sim 120 \%$ stretchability for $R=0.25 \mathrm{~mm}$ and $L=3 \mathrm{~mm}$.

High electric resistances exist in thin buckled interconnects; this inevitably brings high power dissipation and heat generation. Therefore, it is necessary to examine the resistances of the present design. For the serpentine structure under consideration, the resistance per unit apparent length along the direction of stretching, denoted by $R_{\Omega}$ and nondimensionalized by $R w / \rho$, is

$\frac{R w}{\rho} R_{\Omega}=\frac{1}{2 \bar{t}}(\pi+\bar{L})$,

where $\rho$ is resistivity of the interconnect, $w$ is width, $\bar{t}=t / R$ ( $t$ is thickness), and $\bar{L}=L / R$. It is seen that the non-dimensional resistance per unit apparent length of the interconnect depends only on the non-dimensional thickness $\bar{t}$ and nondimensional length $\bar{L}$, as plotted in Figure 4 via $(R w / \rho) R_{\Omega}$ versus $\bar{t}$ for $L=4,8,12$, and 16 , respectively. It is obvious that the resistance per unit apparent length along the direction of stretching is inversely proportional to the thickness. Increase of the thickness from hundreds of nanometers (buckling designs) to dozens of micrometers can reduce the resistance as well as heat generation by two orders of magnitude. On the other hand, longer straight segments yield an
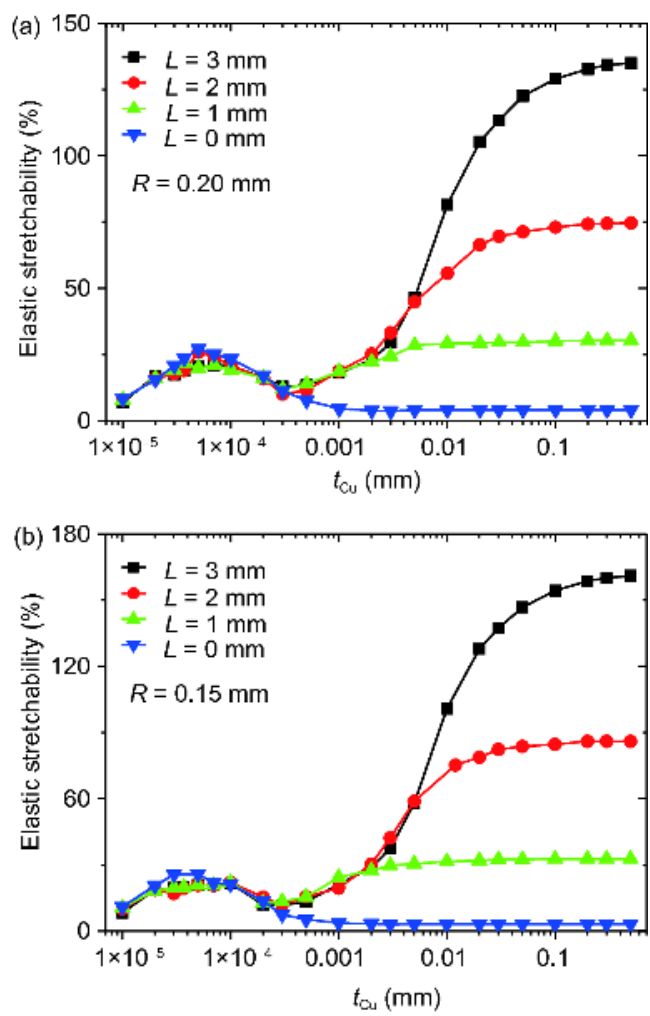

Figure 3 (Color online) Elastic stretchability versus the thickness of $\mathrm{Cu}$ interconnects having (a) $R=0.2 \mathrm{~mm}$ and (b) $R=0.15 \mathrm{~mm}$, with $L=0,1,2$, and $3 \mathrm{~mm}$.

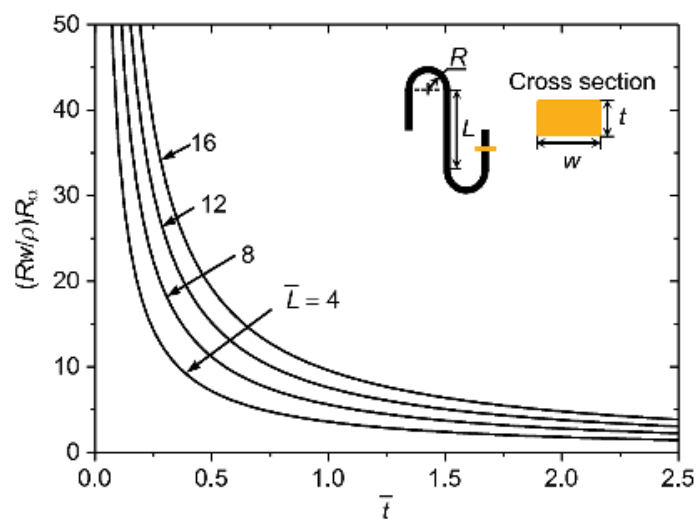

Figure 4 (Color online) Curves of the non-dimensional resistances versus the thicknesses for the interconnects with $\bar{L}=4,8,12$, and 16 .

increase of the resistance, although it increases the stretchability for the nonbuckling design. Therefore, optimization of the designs needs to balance the requirements of both the mechanical and electric performances.

It is concluded from the above analysis that whether the nonbuckling design wins on stretchability depends on the layouts of straight segments and arcs. Basically, long straight segments and small arc radii help to enhance the stretchability, provided that the thicknesses of interconnects are large enough. However, to lower the resistances, shorter straight segments or thicker interconnects are better. Balance 
of the requirements of the mechanical and electric performances should be conducted in practical applications.

\section{Finite deformation mechanics of nonbuckling interconnects}

In this section, we solve the finite deformation problem of a nonbuckling serpentine structure. Accurate deformation configurations during stretching are obtained. The results are of significance for gaining insight into the complex mechanical behavior of the serpentine structure and are useful for refined nonbuckling design of stretchable electronics.

Figure 5(a) illustrates a serpentine structure with multiple periods under horizontal stretching. In view of symmetry, a quarter period of the structure is analyzed, as shown in Figure 5(b) for the initial stress-free state and in Figure 5(c) for the deformed state. The model incorporates a quarter circle with radius $R$ and a straight-line segment with length $L / 2$. Noting that the right end $A$ is an inflection point, the zero-bending moment condition requires that $A$ is simply supported, allowing free rotation and horizontal displacement along the $x$ axis. The left end $C$ is guided, allowing only vertical displacement along the $y$ axis without rotation. The horizontal force $P$ is applied at $A$ due to stretching.

We begin the analysis by giving the governing equation of the structure based on the elastica theory [27]:

$E I \frac{\mathrm{d}\left(\theta-\theta_{0}\right)}{\mathrm{d} s}=P y$,

where $E I$ is the flexural rigidity of the structure, with $E$ being the Young's modulus and $I$ the moment of inertia of cross section; $\theta_{0}$ and $\theta$ are the angles between the $x$ axis and the tangent at a point in the undeformed and deformed configurations, respectively; $s$ is the curvilinear coordinate denoting the distance along the axis of curved structure from point $A$ (Figure 5(c)). Since $\mathrm{d}^{2} \theta_{0} / \mathrm{d} s^{2}=0$ and $\mathrm{d} y / \mathrm{d} s=\sin \theta$, we have $E I \mathrm{~d}^{2} \theta / \mathrm{d} s^{2}=P \sin \theta$ from eq. (2). The integration yields $\frac{1}{2} E I\left(\frac{\mathrm{d} \theta}{\mathrm{d} s}\right)^{2}=-P \cos \theta+C$,

where $C$ is the constant to be determined. In the following, we respectively consider the segments $A B$ and $B C$.

For $A B$, substituting the boundary condition that $\mathrm{d} \theta / \mathrm{d} s=0$ at $A$ into eq. (3) gives $C=P \cos \theta_{A}$, where $\theta_{A}$ is the angle between the $x$ axis and the tangent at $A$ in the deformed configuration. Since $\mathrm{d} \theta / \mathrm{d} s>0$ in $A B$, we obtain $\mathrm{d} s=\mathrm{d} \theta / \sqrt{2 P\left(\cos \theta_{A}-\cos \theta\right) / E I}$ from eq. (3). The length of $A B$ is

$\frac{L}{2}=\int_{0}^{L / 2} \mathrm{~d} s=\int_{\theta_{A}}^{\theta_{B}} \frac{1}{\sqrt{\frac{2 P}{E I}\left(\cos \theta_{A}-\cos \theta\right)}} \mathrm{d} \theta$, where $\theta_{B}$ is the angle between the $x$ axis and the tangent at $B$ in the deformed configuration. The coordinates of a point in $A B$ can be obtained as:

$$
\begin{aligned}
& x=\int_{0}^{s} \cos \theta \mathrm{d} s=\int_{\theta_{A}}^{\theta} \frac{\cos \theta}{\sqrt{\frac{2 P}{E I}\left(\cos \theta_{A}-\cos \theta\right)}} \mathrm{d} \theta, \\
& y=\int_{0}^{s} \sin \theta \mathrm{d} s=\int_{\theta_{A}}^{\theta} \frac{\sin \theta}{\sqrt{\frac{2 P}{E I}\left(\cos \theta_{A}-\cos \theta\right)}} \mathrm{d} \theta .
\end{aligned}
$$

For $B C$, substituting the boundary condition that $\theta=0$ at $C$ into eq. (3) gives $C=P+E I\left(\mathrm{~d} \theta /\left.\mathrm{d} s\right|_{C}\right)^{2} / 2$. Since $\mathrm{d} \theta / \mathrm{d} s<0$ in $B C$, we obtain $\mathrm{d} s=-\mathrm{d} \theta / \sqrt{2 P(1-\cos \theta) / E I+\left(\mathrm{d} \theta /\left.\mathrm{d} s\right|_{C}\right)^{2}}$ from eq. (3). The length of $B C$ is

$$
\frac{\pi R}{2}=-\int_{\theta_{B}}^{0} \frac{1}{\sqrt{\frac{2 P}{E I}(1-\cos \theta)+\left(\left.\frac{\mathrm{d} \theta}{\mathrm{d} s}\right|_{C}\right)^{2}}} \mathrm{~d} \theta .
$$

The coordinates of a point in $B C$ can be obtained as:

$$
\begin{aligned}
x= & \int_{\theta_{A}}^{\theta_{B}} \frac{\cos \theta}{\sqrt{\frac{2 P}{E I}\left(\cos \theta_{A}-\cos \theta\right)}} \mathrm{d} \theta \\
& -\int_{\theta_{B}}^{\theta} \frac{\cos \theta}{\sqrt{\frac{2 P}{E I}(1-\cos \theta)+\left(\left.\frac{\mathrm{d} \theta}{\mathrm{d} s}\right|_{C}\right)^{2}}} \mathrm{~d} \theta, \\
y= & \int_{\theta_{A}}^{\theta_{B}} \frac{\sin \theta}{\sqrt{\frac{2 P}{E I}\left(\cos \theta_{A}-\cos \theta\right)}} \mathrm{d} \theta \\
& -\int_{\theta_{B}}^{\theta} \frac{\sin \theta}{\sqrt{\frac{2 P}{E I}(1-\cos \theta)+\left(\left.\frac{\mathrm{d} \theta}{\mathrm{d} s}\right|_{C}\right)^{2}}} \mathrm{~d} \theta .
\end{aligned}
$$

The horizontal and vertical distances from $A$ to $C$, i.e., $x_{C}$ and $y_{C}$, are respectively obtained as:

$$
\begin{aligned}
x_{C}= & \int_{\theta_{A}}^{\theta_{B}} \frac{\cos \theta}{\sqrt{\frac{2 P}{E I}\left(\cos \theta_{A}-\cos \theta\right)}} \mathrm{d} \theta \\
& +\int_{0}^{\theta_{B}} \frac{\cos \theta}{\sqrt{\frac{2 P}{E I}(1-\cos \theta)+\left(\left.\frac{\mathrm{d} \theta}{\mathrm{d} s}\right|_{C}\right)^{2}}} \mathrm{~d} \theta,
\end{aligned}
$$

and 


$$
\begin{aligned}
y_{C}= & \int_{\theta_{A}}^{\theta_{B}} \frac{\sin \theta}{\sqrt{\frac{2 P}{E I}\left(\cos \theta_{A}-\cos \theta\right)}} \mathrm{d} \theta \\
& +\int_{0}^{\theta_{B}} \frac{\sin \theta}{\sqrt{\frac{2 P}{E I}(1-\cos \theta)+\left(\left.\frac{\mathrm{d} \theta}{\mathrm{d} s}\right|_{C}\right)^{2}}} \mathrm{~d} \theta .
\end{aligned}
$$

At $B$, the difference of curvatures derived from $A B$ and $B C$ remains unchanged before and after deformation; this leads to

$$
\begin{aligned}
& \sqrt{\frac{2 P}{E I}\left(\cos \theta_{A}-\cos \theta_{B}\right)} \\
& +\sqrt{\frac{2 P}{E I}\left(1-\cos \theta_{B}\right)+\left(\left.\frac{\mathrm{d} \theta}{\mathrm{d} s}\right|_{C}\right)^{2}}=\frac{1}{R} .
\end{aligned}
$$

Introducing $\bar{R}=\sqrt{2 P / E I} R, \bar{\theta}_{C}^{\prime}=\sqrt{E I / 2 P} \mathrm{~d} \theta /\left.\mathrm{d} s\right|_{C}$, and
$\bar{x}_{C}=x_{C} / R$, recalling $L=L / R$, the following equations are obtained from eqs. (4), (6), (8), and (10):

$$
\left\{\begin{array}{l}
\int_{\theta_{A}}^{\theta_{B}} \frac{1}{\sqrt{\cos \theta_{A}-\cos \theta}} \mathrm{d} \theta=\frac{\bar{R} \bar{L}}{2}, \\
-\int_{\theta_{B}}^{0} \frac{1}{\sqrt{1-\cos \theta+\bar{\theta}^{\prime}{ }_{C}^{2}}} \mathrm{~d} \theta=\frac{\pi \bar{R}}{2}, \\
\sqrt{\left(\cos \theta_{A}-\cos \theta_{B}\right)}+\sqrt{\left(1-\cos \theta_{B}\right)+\bar{\theta}_{C}^{\prime}{ }^{2}}=\frac{1}{\bar{R}}, \\
\int_{\theta_{A}}^{\theta_{B}} \frac{\cos \theta}{\sqrt{\cos \theta_{A}-\cos \theta}} \mathrm{d} \theta+\int_{0}^{\theta_{B}} \frac{\cos \theta}{\sqrt{1-\cos \theta+\bar{\theta}_{C}^{\prime}}} \mathrm{d} \theta=\bar{R} \bar{x}_{C} .
\end{array}\right.
$$

At a given horizontal displacement in stretching, i.e., specifying $\bar{x}_{C}$, one can obtain $\bar{R}, \theta_{A}, \theta_{B}$, and $\bar{\theta}^{\prime}{ }_{C}$ from the above set of equations via the commercial software package MATLAB. The coordinates of any point in the structure (a)

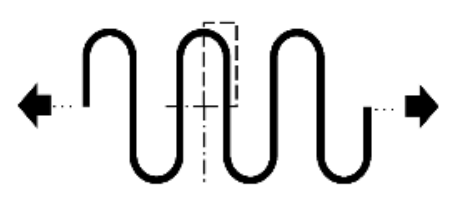

(b)

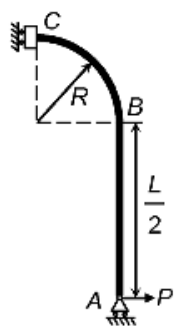

(c)

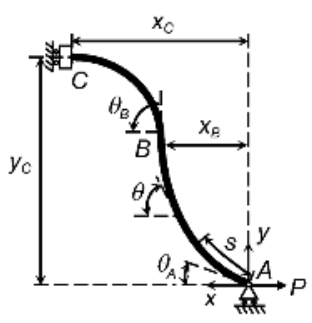

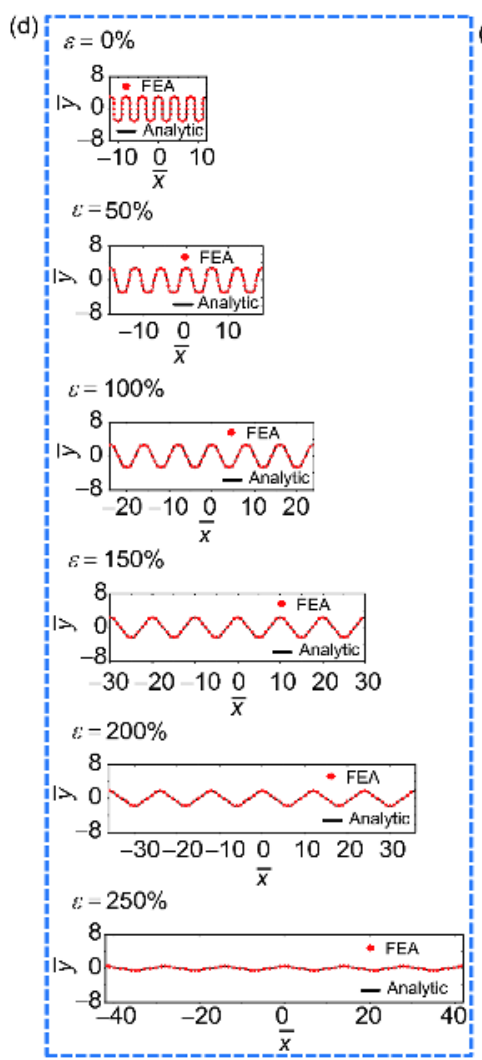
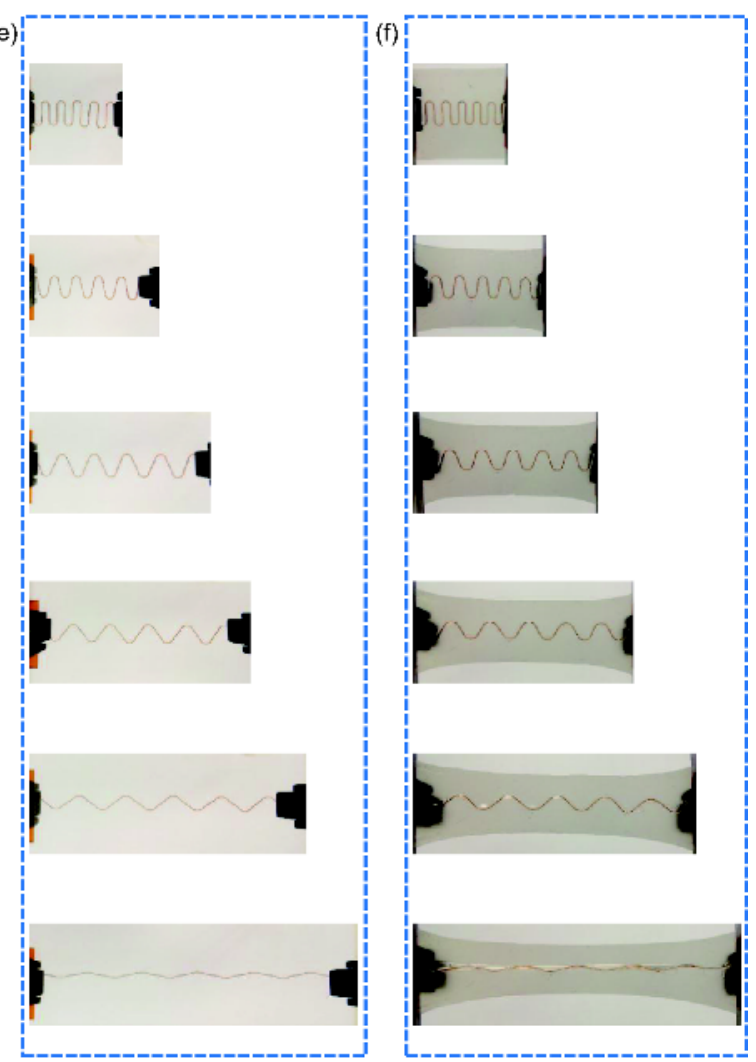

Figure 5 (Color online) (a) Serpentine interconnect with multiple periods under horizontal stretching. A quarter period of the interconnect in (b) the initial stress-free state and (c) deformed state. (d) Comparison of the configurations between the analytic model and FEA under different applied strains. Experimental configurations with the interconnect being (e) freestanding or (f) bonded to an Ecoflex substrate. 
during stretching are then obtained by eqs. (5) and (7).

The accuracy of the analytic results is confirmed by perfect agreement with FEA, as shown in Figure 5(d), in which $\bar{x}=x / R$ and $\bar{y}=y / R$. A more refined set of configurations of the interconnects with different lengths of straight segments are plotted in Figure al (see Appendix for details), where the accuracy of the analytic model is well validated again. To further test the applicability of our model, we fabricate two specimens of PI interconnects $(R=0.8 \mathrm{~mm}$, $t=w=0.1 \mathrm{~mm}$, and $L=3.2 \mathrm{~mm}$ ), with one being freestanding (Figure 5(e)) and the other one bonded to a $0.5-\mathrm{mm}$ thick Ecoflex substrate (Figure 5(f)). Six periods are taken to eliminate the boundary effects of the specimens, which are quasi-statically stretched to $350 \%$ applied strain in a microtensile material-testing machine (Instron 5848 Microtester). The recorded configurations during stretching include those unstretched and those stretched to $50 \%, 100 \%, 150 \%, 200 \%$, and $250 \%$ applied strains, respectively. All the experimental results agree very well with both FEA and our analytic model, regardless of the Ecoflex substrate; this also confirms the negligible effect of the substrate, i.e., encapsulation has negligible impact on the deformation mode and stretchability of nonbuckling interconnects, which is an important basis for feasibility of nonbuckling-based stretchable electronics. The force-displacement relationship is also experimentally obtained, which shows favorable agreement with the analytic finding, as reflected in Figure 6(a) for the curve of $P$ versus the applied strain $\bar{x}_{C}-1$.

The maximum strain in an interconnect is reached at $C$, where the maximum bending moment is reached. We have

$\varepsilon_{\max }=\frac{w}{2} \Delta \kappa=\frac{w}{2}\left(\frac{1}{R}+\left.\frac{\mathrm{d} \theta}{\mathrm{d} s}\right|_{C}\right)$,

where $\Delta \kappa$ is the curvature change from the initial configuration. Eq. (12) holds under the following conditions: (1) the material maintains a linear-elastic state during deformation, and (2) the membrane strain is much small than the bending strain. Accordingly, eq. (12) implies two extreme cases: (1) $\mathrm{d} \theta /\left.\mathrm{d} s\right|_{C}=-1 / R$, resulting in $\varepsilon_{\max }=0$, indicating the initial stress-free configuration, and (2) $\mathrm{d} \theta /\left.\mathrm{d} s\right|_{C}=0$, resulting in $\varepsilon_{\max }=w /(2 R)$, indicating straightening of the interconnect. At a given applied strain $\bar{x}_{C}-1, R \mathrm{~d} \theta /\left.\mathrm{d} s\right|_{C}$ can be obtained by eq. (11), substitution of which into the nondimensional form of eq. (12), $R \varepsilon_{\max } / w=\left(1+R \mathrm{~d} \theta /\left.\mathrm{d} s\right|_{C}\right) / 2$, gives the curve of the maximum interconnect strain versus the applied strain, as shown in Figure 6(b) for $\bar{L}=4,8,12$, and 16 , respectively. To quantitatively characterize how easy the interconnects with different lengths of straight segments are stretched to specified applied strains, the non-dimensional force-applied strain curves corresponding to Figure 6(b) are plotted in Figure 6(c). It is seen from Figure 6(b) and (c) that both the maximum strain and applied force increase with
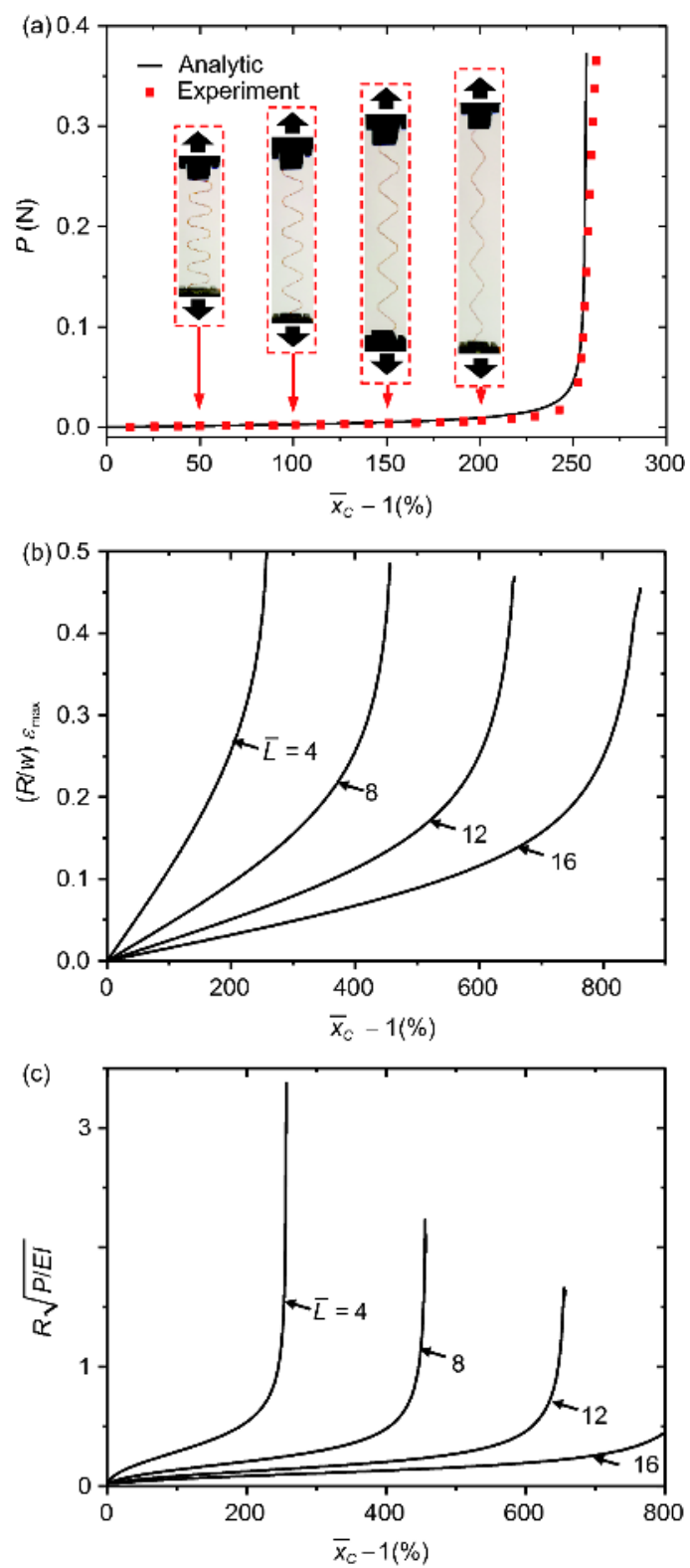

Figure 6 (Color online) (a) Comparison of the force-applied strain relationships of the freestanding interconnect between the analytic and experimental results; (b) curves of the non-dimensional maximum strains versus the applied strains; (c) curves of the non-dimensional forces versus the applied strains for the interconnects with $\bar{L}=4,8,12$, and 16 .

the applied strain, as one can expect. On the other hand, with the increase of $\bar{L}$, both the maximum strain and applied force decrease, which indicates that higher stretchability and easier stretching can be simultaneously accomplished by increasing the lengths of straight segments of the interconnects.

\section{Conclusions}

The nonbuckling design offers a novel approach to stretchable electronics with high elastic stretchability, easiness for encapsulation, and high electric performance. By compre- 
hensive analytic, numerical and experimental investigations, the following conclusions are drawn.

(1) A significant finding is quantitatively revealed that whether the nonbuckling interconnects win on stretchability depends on the layouts.

(2) An important design strategy is proposed that long straight segments and small arc radii for nonbuckling interconnects should be taken for highly stretchable electronics.

(3) The analysis of electric resistances suggests that, to lower the resistances, shorter straight segments or thicker interconnects are better. Therefore, optimization of the designs needs to balance the requirements of both the mechanical and electric performances.

(4) An analytic finite deformation model is established, and accurate mechanical behaviors are obtained for nonbuckling interconnects under stretching, which is of significance for the design of nonbuckling-based stretchable electronics.

This work was supported by the National Natural Science Foundation of China (Grant Nos. 11572323, 11772331, 11302038, 51365013, and 11732004), the Chinese Academy of Sciences via the "Hundred Talent Program", the Strategic Priority Research Program of the Chinese Academy of Sciences (Grant No. XDB22040501), the State Key Laboratory of Structural Analysis for Industrial Equipment, Dalian University of Technology (Grant No. GZ1603), the State Key Laboratory of Digital Manufacturing Equipment and Technology, Huazhong University of Science and Technology (Grant No. DMETKF2017008), the Young Elite Scientists Sponsorship Program by CAST (Grant No. 2015QNRC001), the Opening Fund of State Key Laboratory of Nonlinear Mechanics, the Personnel Training Plan of Tianjin City in China for the Key Young and Middle-Aged Innovation Talents, the National Key Research and Development Plan (Grant Nos. 2016YFB0201600, 2016YFB0201601, 2017YFB0202800, and 2017YFB0202802), the Program for Changjiang Scholars, Innovative Research Team in University (PCSIRT), and the 111 Project (Grant No. B14013).

1 J. A. Rogers, T. Someya, and Y. Huang, Science 327, 1603 (2010).

2 H. C. Ko, M. P. Stoykovich, J. Song, V. Malyarchuk, W. M. Choi, C. J. Yu, J. B. Geddes III, J. Xiao, S. Wang, Y. Huang, and J. A. Rogers, Nature 454, 748 (2008).

3 Y. M. Song, Y. Xie, V. Malyarchuk, J. Xiao, I. Jung, K. J. Choi, Z. Liu, H. Park, C. Lu, R. H. Kim, R. Li, K. B. Crozier, Y. Huang, and J. A. Rogers, Nature 497, 95 (2013).

4 D. H. Kim, N. Lu, R. Ma, Y. S. Kim, R. H. Kim, S. Wang, J. Wu, S. M. Won, H. Tao, A. Islam, K. J. Yu, T. Kim, R. Chowdhury, M. Ying, L. $\mathrm{Xu}$, M. Li, H. J. Chung, H. Keum, M. McCormick, P. Liu, Y. W. Zhang, F. G. Omenetto, Y. Huang, T. Coleman, and J. A. Rogers, Science 333, 838 (2011).

5 M. Ying, A. P. Bonifas, N. Lu, Y. Su, R. Li, H. Cheng, A. Ameen, Y. Huang, and J. A. Rogers, Nanotechnology 23, 344004 (2012).

6 Y. Su, R. Li, H. Cheng, M. Ying, A. P. Bonifas, K. C. Hwang, J. A. Rogers, and Y. Huang, J. Appl. Phys. 114, 164511 (2013).

7 S. Xu, Y. Zhang, J. Cho, J. Lee, X. Huang, L. Jia, J. A. Fan, Y. Su, J. Su, H. Zhang, H. Cheng, B. Lu, C. Yu, C. Chuang, T. I. Kim, T. Song, K. Shigeta, S. Kang, C. Dagdeviren, I. Petrov, P. V. Braun, Y. Huang, U. Paik, and J. A. Rogers, Nat. Commun. 4, 1543 (2013).

8 N. Lu, and D. H. Kim, Soft Robotics 1, 53 (2014).

9 D. Y. Khang, H. Jiang, Y. Huang, and J. A. Rogers, Science 311, 208 (2006).
10 W. M. Choi, J. Song, D. Y. Khang, H. Jiang, Y. Y. Huang, and J. A. Rogers, Nano Lett. 7, 1655 (2007).

11 D. H. Kim, J. Song, W. Mook Choi, H. S. Kim, R. H. Kim, Z. Liu, Y. Y. Huang, K. C. Hwang, Y. Zhang, and J. A. Rogers, Proc. Natl. Acad. Sci. USA 105, 18675 (2008).

12 R. H. Kim, D. H. Kim, J. Xiao, B. H. Kim, S. I. Park, B. Panilaitis, R. Ghaffari, J. Yao, M. Li, Z. Liu, V. Malyarchuk, D. G. Kim, A. P. Le, R. G. Nuzzo, D. L. Kaplan, F. G. Omenetto, Y. Huang, Z. Kang, and J. A. Rogers, Nat. Mater. 9, 929 (2010).

13 D. H. Kim, N. Lu, Y. Huang, and J. A. Rogers, MRS Bull. 37, 226 (2012).

14 S. Xu, Y. Zhang, L. Jia, K. E. Mathewson, K. I. Jang, J. Kim, H. Fu, X. Huang, P. Chava, R. Wang, S. Bhole, L. Wang, Y. J. Na, Y. Guan, M. Flavin, Z. Han, Y. Huang, and J. A. Rogers, Science 344, 70 (2014).

15 J. A. Fan, W. H. Yeo, Y. Su, Y. Hattori, W. Lee, S. Y. Jung, Y. Zhang, Z. Liu, H. Cheng, L. Falgout, M. Bajema, T. Coleman, D. Gregoire, R. J. Larsen, Y. Huang, and J. A. Rogers, Nat. Commun. 5, 3266 (2014).

16 Y. Su, S. Wang, Y. A. Huang, H. Luan, W. Dong, J. A. Fan, Q. Yang, J. A. Rogers, and Y. Huang, Small 11, 367 (2015).

17 Y. Su, X. Ping, K. J. Yu, J. W. Lee, J. A. Fan, B. Wang, M. Li, R. Li, D. V. Harburg, Y. A. Huang, C. Yu, S. Mao, J. Shim, Q. Yang, P. Y. Lee, A. Armonas, K. J. Choi, Y. Yang, U. Paik, T. Chang, T. J. Dawidczyk, Y. Huang, S. Wang, and J. A. Rogers, Adv. Mater. 29, 1604989 (2017).

18 S. Xu, Z. Yan, K. I. Jang, W. Huang, H. Fu, J. Kim, Z. Wei, M. Flavin, J. McCracken, R. Wang, A. Badea, Y. Liu, D. Xiao, G. Zhou, J. Lee, H. U. Chung, H. Cheng, W. Ren, A. Banks, X. Li, U. Paik, R. G. Nuzzo, Y. Huang, Y. Zhang, and J. A. Rogers, Science 347, 154 (2015).

19 Y. Zhang, S. Xu, H. Fu, J. Lee, J. Su, K. C. Hwang, J. A. Rogers, and Y. Huang, Soft Matter 9, 8062 (2013).

20 H. Fu, S. Xu, R. Xu, J. Jiang, Y. Zhang, J. A. Rogers, and Y. Huang, Appl. Phys. Lett. 106, 091902 (2015).

21 L. Liu, and N. Lu, Int. J. Solids Struct. 87, 48 (2016).

22 Y. Zhang, S. Wang, X. Li, J. A. Fan, S. Xu, Y. M. Song, K. J. Choi, W. H. Yeo, W. Lee, S. N. Nazaar, B. Lu, L. Yin, K. C. Hwang, J. A. Rogers, and Y. Huang, Adv. Funct. Mater. 24, 2028 (2014).

23 T. Widlund, S. Yang, Y. Y. Hsu, and N. Lu, Int. J. Solids Struct. 51, 4026 (2014).

24 Z. Fan, Y. Zhang, Q. Ma, F. Zhang, H. Fu, K. C. Hwang, and Y. Huang, Int. J. Solids Struct. 91, 46 (2016).

25 S. Yang, S. Qiao, and N. Lu, J. Appl. Mech. 84, 021004 (2016).

26 ABAQUS. 2013 Analysis User's Guide V6.13 (Dassault Systèmes, Pawtucket, RI, 2013).

27 S. P. Timoshenko, and Gere J. M. Gere, Theory of Elastic Stability (McGraw-Hill Company, New York, 1961).

\section{Appendix Validation of analytic configura- tion solutions}

To check the accuracy of the analytic model for the interconnect configurations during stretching, we conducted FEA with the ABAQUS software package [26], where the linear beam element was adopted for a PI serpentine interconnect with $E=2.5 \mathrm{GPa}, R=0.25 \mathrm{~mm}, t=w=50 \mu \mathrm{m}$ (yielding $\left.I=5.208 \times 10^{-7} \mathrm{~mm}^{4}\right), L=1 \mathrm{~mm}, 2 \mathrm{~mm}, 3 \mathrm{~mm}$, and $4 \mathrm{~mm}$, respectively. Figure a1(a)-(d) shows the deformation configurations of a quarter period and Figure a1(e)-(h) shows those of a complete period, with $\bar{L}=4,8,12$, and 16 , respectively. Three representative configurations during stretching have been demonstrated for each case. It is obvious that all the analytic results agree very well with their counterparts from FEA. 

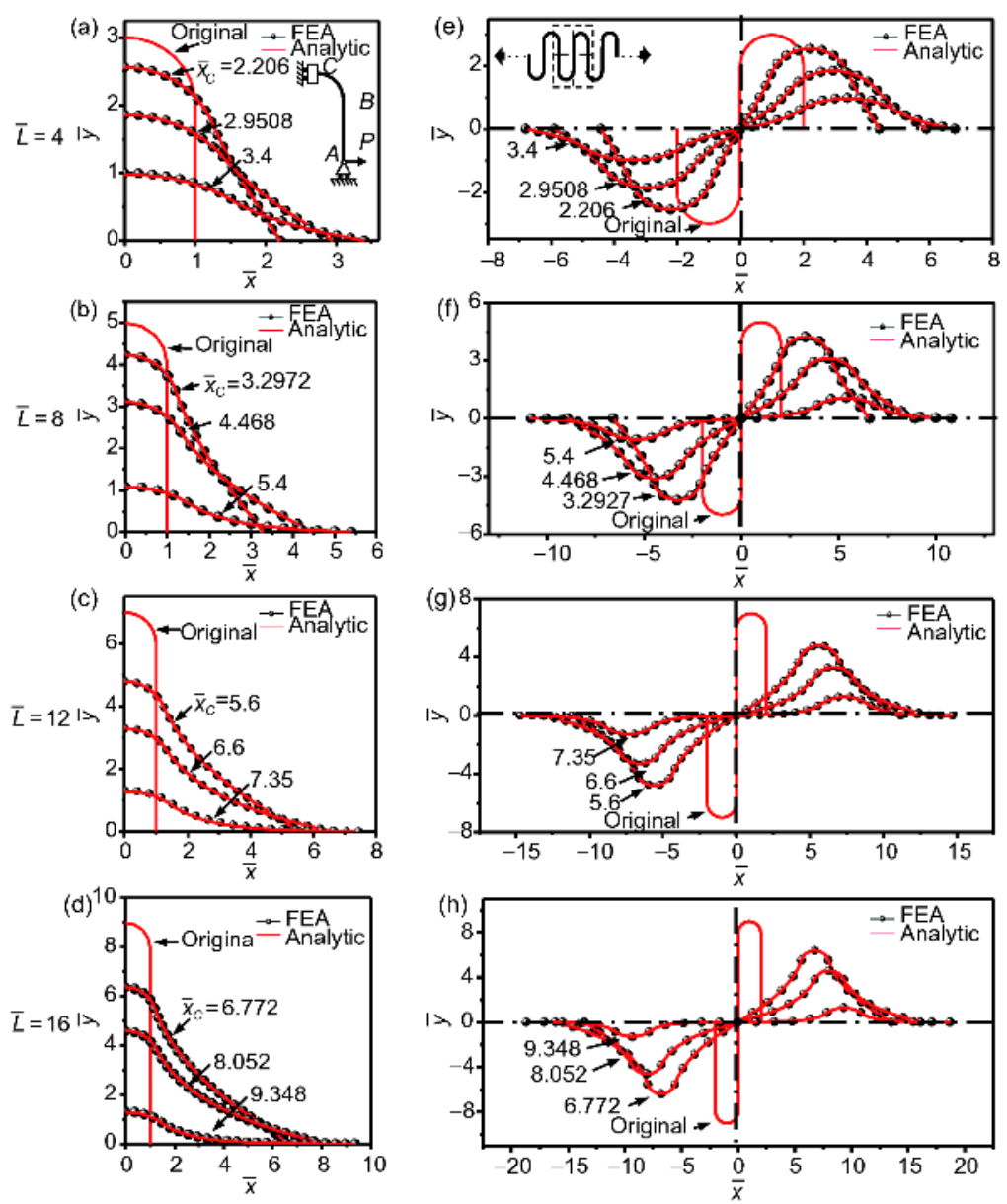

Figure a1 (Color online) Comparison of the configurations of stretched interconnects between the analytic model and FEM. (a)-(d) A quarter period of the interconnects with $\bar{L}=4,8,12$, and 16 ; (e)-(h) a complete period of the interconnects corresponding to (a)-(d). 